\title{
Entropic release of a big sphere from a cylindrical vessel
}

\section{$\operatorname{AUTHOR}(\mathrm{S})$ :}

Mishima, Hirokazu; Oshima, Hiraku; Yasuda, Satoshi; Amano, Ken-ichi; Kinoshita, Masahiro

\section{CITATION:}

Mishima, Hirokazu ... [et al]. Entropic release of a big sphere from a cylindrical vessel. Chemical Physics Letters 2013, 561-562: 159-165

\section{ISSUE DATE:}

2013-03

URL:

http://hdl.handle.net/2433/172456

\section{RIGHT:}

(C) 2013 Elsevier B.V.; This is not the published version. Please cite only the published version.; この論文は出版社版でありません。引用の際に は出版社版をご確認ご利用ください。 


\title{
Entropic release of a big sphere from a cylindrical vessel
}

\author{
Hirokazu Mishima ${ }^{1}$, Hiraku Oshima ${ }^{2}$, Satoshi Yasuda ${ }^{1}$, Ken-ichi Amano ${ }^{3}$, \\ Masahiro Kinoshita ${ }^{2}$ \\ ${ }^{1}$ Graduate School of Energy Science, Kyoto University, Uji, Kyoto 611-0011, Japan \\ ${ }^{2}$ Institute of Advanced Energy, Kyoto University, Uji, Kyoto 611-0011, Japan \\ ${ }^{3}$ Graduate School of Science, Kobe University, Nada, Kobe, Hyogo 657-8501, Japan
}

Author to whom correspondence should be addressed: Masahiro Kinoshita.

E-mail address: kinoshit@iae.kyoto-u.ac.jp.

\begin{abstract}
Insertion and release of a solute into and from a cylindrical vessel comprising biopolymers is a fundamental function in biological systems. In earlier works, we reported that the solvent-entropy (SE) effect plays imperative roles for insertion. Here we show that release is also achievable by the SE effect: The solute can be moved from an entrance at one end of the vessel to an exit at the other end using a continuous variation of the vessel geometry. Since the SE effect is rather insensitive to the solute-solvent affinity, our result may provide a clue to the "multidrug efflux" of TolC.
\end{abstract}




\section{Introduction}

Insertion of a solute into a vessel comprising biopolymers followed by release of the same solute from it is a fundamental function in biological systems. It is quite interesting that the two apparently opposite events, insertion and release, successively occur in a system. A typical example of such an insertion/release process is found for TolC: an important component of the tripartite efflux system, AcrA/AcrB/TolC [1-3]. AcrB is an efflux pump protein, TolC is a cylindrical vessel possessing an entrance at one end and an exit at the other end for the solute, and AcrA mediates the contact between AcrB and TolC. AcrB interacting with TolC at the entrance sends the solute to the central position within the vessel cavity of TolC, and then the solute is moved to the exit.

In earlier works [4-6], we showed that the solute-vessel potential of mean force (PMF denoted by $\Phi$ ) formed by the solvent plays imperative roles for insertion. The PMF represents "the free energy of the solvent for a fixed configuration of the solute-vessel pair" minus "that for the configuration where the solute is infinitely far from the vessel". It is physically insightful to decompose the PMF scaled by $k_{\mathrm{B}} T\left(k_{\mathrm{B}}\right.$ is Boltzmann's constant and $T$ is the absolute temperature $), \Phi /\left(k_{\mathrm{B}} T\right)$, into its energetic and entropic components denoted by $\Phi_{\mathrm{E}} /\left(k_{\mathrm{B}} T\right)$ and $-\Phi_{\mathrm{S}} / k_{\mathrm{B}}$, respectively: $\Phi /\left(k_{\mathrm{B}} T\right)=\Phi_{\mathrm{E}} /\left(k_{\mathrm{B}} T\right)-\Phi_{\mathrm{S}} / k_{\mathrm{B}}$.

A target of our earlier works was the protein flux through a chaperonin system [5-9]: An unfolded protein is inserted into the chaperonin from bulk aqueous solution, protein folding occurs within the chaperonin cavity, and the folded protein is released back to the bulk. The conclusions drawn were as follows $[5,6]$.

(1) $-\Phi_{\mathrm{S}} / k_{\mathrm{B}}$ represents the solvent-entropy effect originating from the translational displacement of solvent molecules. It is closely related to the excluded volume (EV) generated by the solute. (The solute generates a space which the centers of solvent molecules cannot enter, and the volume of this space is the EV.) $-\Phi_{\mathrm{S}} / k_{\mathrm{B}}$ is rather insensitive to the solute-solvent and vessel inner surface-solvent affinities, namely, to whether the solute or the vessel inner surface is solvophobic or solvophilic (on condition that they are neither too solvophobic nor too solvophilic). $-\Phi_{\mathrm{S}} / k_{\mathrm{B}}$ always drives the solute to be inserted into the vessel cavity and constrained within a small space almost in the center. The power of insertion and constraint becomes stronger as the EV increases.

(2) $\Phi_{\mathrm{E}} /\left(k_{\mathrm{B}} T\right)$ is strongly dependent on the solute-solvent and vessel inner surface-solvent affinities. The roles of $\Phi_{\mathrm{E}} /\left(k_{\mathrm{B}} T\right)$ can be summarized as follows. When the inner surface of the vessel is solvophilic, the solvent number density within the vessel cavity is higher than that in the bulk aqueous solution. As a result, a solvophilic solute is preferentially solvated within the cavity, whereas a solvophobic solute is more stabilized in the bulk solution. When the inner surface is solvophobic, the solvent number density within the cavity is lower than that in the bulk. Consequently, a 
solvophobic solute is more stabilized within the cavity, whereas a solvophilic solute is preferentially solvated in the bulk.

(3) As for the protein flux through a chaperonin system, when a protein is inserted or released, the inner surface of chaperonin is weakly hydrophobic [5-9]. An unfolded protein with large EV and weak hydrophobicity is driven to be weakly inserted by $\Phi_{\mathrm{E}} /\left(k_{\mathrm{B}} T\right)$ and strongly inserted by $-\Phi_{\mathrm{S}} / k_{\mathrm{B}}$, and the net action is insertion. The switch from insertion to release is achieved by altering the protein properties as follows. The folding reduces the EV and makes the protein more hydrophilic. $-\Phi_{\mathrm{S}} / k_{\mathrm{B}}$ and the power of insertion then become weaker, with the result that $\Phi_{\mathrm{E}} /\left(k_{\mathrm{B}} T\right)$ dominates. The folded protein is released back to the bulk aqueous solution to become preferentially hydrated in the bulk. The switch from insertion to release is thus realized, and $-\Phi_{\mathrm{S}} / k_{\mathrm{B}}$ drives insertion while $\Phi_{\mathrm{E}} /\left(k_{\mathrm{B}} T\right)$ is requisite in release.

In TolC, by contrast, the solute properties remain unchanged for insertion and release, and the switch from insertion to release should occur through a different mechanism. AcrA/AcrB/TolC extrudes a variety of drug molecules (i.e., nonpolar, polar, and charged solutes), and this variability is known as "multidrug efflux" [1-3]. However, its microscopic mechanism remains rather mysterious and has not been elucidated yet. We emphasize that the multidrug efflux of AcrA/AcrB/TolC can be performed only when TolC as well as AcrB exhibits this feature. In this Letter, we are concerned with the multidrug efflux of TolC. The multidrug efflux is indicative that its mechanism cannot be chemically specific but rather has to be based on a physical factor. We show that the factor is a time-dependent entropic force acting on a solute, which originates from the solvent-mediated interaction between the solute and TolC whose geometry is time dependent. The model system employed is very simple but takes this important factor into consideration.

On the basis of the results summarized in (2), if $\Phi_{\mathrm{E}} /\left(k_{\mathrm{B}} T\right)$ dominates, we can conclude the following. In cases where the inner surface of the vessel is hydrophilic, only hydrophilic solutes are inserted, and they are released when the inner surface turns hydrophobic. In cases where the inner surface is hydrophobic, only hydrophobic solutes are inserted, and they are released when the inner surface turns hydrophilic. It is thus difficult to treat both of hydrophilic and hydrophobic solutes: The multidrug efflux cannot be exhibited if $\Phi_{\mathrm{E}} /\left(k_{\mathrm{B}} T\right)$ dominates. To realize the multidrug efflux, the PMF must be governed by $-\Phi_{\mathrm{S}} / k_{\mathrm{B}}$ with the insensitivity mentioned in (1). An important point is that when the vessel inner surface is neither hydrophilic nor hydrophobic and the solute within the cavity and that in the bulk aqueous solution share almost the same stability, $\Phi_{\mathrm{E}} /\left(k_{\mathrm{B}} T\right)$ is much less powerful than $-\Phi_{\mathrm{S}} / k_{\mathrm{B}}$ (i.e., $-\Phi_{\mathrm{S}} / k_{\mathrm{B}}$ dominates). This can be relevant to TolC whose inner surface possesses a mixture of predominantly nonpolar and isolated electronegative patches [1]. However, $-\Phi_{\mathrm{S}} / k_{\mathrm{B}}$ always acts for insertion. A question then arises: Can a solute be released without adjusting $\Phi_{\mathrm{E}} /\left(k_{\mathrm{B}} T\right)$ unlike in the chaperonin case?

Here we explore the possibility of releasing the solute by $-\Phi_{\mathrm{S}} / k_{\mathrm{B}}$ alone. We 
calculate the spatial distribution of the PMF between a big sphere and a cylindrical vessel with open ends immersed in small spheres using the three-dimensional (3D) integral equation theory [4-6,10-15] combined with rigid-body models. With the rigid-body models, all of the allowed system configurations share the same energy and the system behavior is purely entropic in origin [15]. Namely, $\Phi /\left(k_{\mathrm{B}} T\right)$ possesses only the entropic component: $\Phi /\left(k_{\mathrm{B}} T\right)=-\Phi_{\mathrm{S}} / k_{\mathrm{B}}$. Unlike in our earlier works, the vessel geometry is made variable after the solute insertion. It is demonstrated that release can also be achieved by $-\Phi_{\mathrm{S}} / k_{\mathrm{B}}$ : The solute is entropically moved from the entrance to the exit by a continuous variation of the vessel geometry. Since the spatial distribution of the PMF becomes largely positive and largely negative with the periodicity of the molecular diameter of the solvent, $d_{\mathrm{S}}=0.28 \mathrm{~nm}$, even a variation of the vessel geometry within the scale of $d_{\mathrm{S}}$ leads to a drastic change in the spatial distribution, thus enabling the vessel to control the solute motion.

In biological systems, the solvent is water characterized by hydrogen bonds. However, in hydration thermodynamics of a solute, the translational entropy predominates over the rotational entropy [16]. The entropic effect originating from the translational displacement of water molecules can be described by modeling water as hard spheres except when its temperature dependence plays essential roles (e.g., in the elucidation of cold denaturation of a protein $[17,18]$ ), as long as the diameter and number density of the hard-sphere solvent are set at those of water [19-21]. We note that the hydrogen-bonding allows water to exist as a dense liquid despite its quite a small molecular size, leading to an exceptionally large entropic effect [20,21].

In AcrA/AcrB/TolC, the proton motive force causes structural changes of AcrB which are transmitted to TolC through AcrA [1-3]. This action may lead to a continuous vessel-geometry variation of TolC. A molecular dynamics (MD) simulation study [2] has also suggested that TolC can vary its geometric characteristics (e.g., they have observed a peristaltic motion of the periplasmic domain). However, the details of the variation during the insertion/release process are still unknown. What we emphasize in this Letter is that release as well as insertion can be accomplished by $-\Phi_{\mathrm{S}} / k_{\mathrm{B}}$ which is rather insensitive to the solute-solvent and vessel inner surface-solvent affinities and that a continuous vessel-geometry variation is a key factor. This result may provide us with a clue to the microscopic mechanism of the multidrug efflux of TolC, pending further studies.

\section{Model and Theory}

$-T \Phi_{\mathrm{S}}$ considered in this Letter is essentially the so-called depletion potential whose physical essence was first discussed by the Asakura-Oosawa theory $[22,23]$. The depletion potential between big bodies with simple geometries (e.g., big spheres, a big sphere and a convex, planer, or concave surface, etc.) [24-28] or between big, nonspherical bodies [13,29,30] has been analyzed using more advanced theories. A feature of the present study is that one of the big bodies possesses a complex geometry 


\section{[4-6,12-15].}

We consider a big vessel and a big sphere immersed at infinite dilution in small spheres with diameter $d_{\mathrm{S}}$ forming the solvent. The big sphere, whose diameter is denoted by $d_{\mathrm{B}}$, corresponds to a solute. The initial geometry of the vessel is illustrated in Fig. 1a. After the solute insertion, the vessel geometry is varied for the solute release as explained in Fig. 1b-f. The Cartesian coordinate system is chosen as illustrated in Fig. 1a. The cross section of $z=0$ is shown for each geometry. More details are described in a later paragraph.

The vessel with a prescribed geometry is considered. First, the vessel-solvent correlation functions are calculated using the 3D integral equation theory [4,6,10-15] described below. Second, the solute-solvent correlation functions are calculated using the radial-symmetric HNC theory [25] for spherical particles. The PMF between the vessel and the solute is then calculated by assuming that the solvent particles are always in equilibrium with each configuration of the vessel-solute pair $[6,14]$.

The Ornstein-Zernike (OZ) equation in the Fourier space is expressed by

$W_{1 \mathrm{~S}}\left(k_{x}, k_{y}, k_{z}\right)=\rho_{\mathrm{S}} C_{1 \mathrm{~S}}\left(k_{x}, k_{y}, k_{z}\right) H_{\mathrm{SS}}(k)$

and the hypernetted-chain (HNC) closure equation is written as

$c_{1 \mathrm{~S}}(x, y, z)=\exp \left\{-u_{1 \mathrm{~S}}(x, y, z) /\left(k_{\mathrm{B}} T\right)\right\} \exp \left\{w_{1 \mathrm{~S}}(x, y, z)\right\}-w_{1 \mathrm{~S}}(x, y, z)-1$.

Here, the subscripts " 1 " and "S" denote the vessel and the solvent, respectively, $w=h-c$, $c$ is the direct correlation function, $h$ the total correlation function, $u$ the potential, and $\rho_{\mathrm{S}}$ the bulk density. The molecular diameter $d_{\mathrm{S}}$ is set at the value of water, $0.28 \mathrm{~nm}$. The reduced number density $\rho_{\mathrm{S}} d_{\mathrm{S}}{ }^{3}$ is taken to be the value of water at $298 \mathrm{~K}$ and $1 \mathrm{~atm}$, 0.7317. The capital letters $\left(C, H\right.$, and $W$ ) represent the Fourier transforms. $H_{\mathrm{SS}}(k)$ $\left(k^{2}=k_{x}{ }^{2}+k_{y}{ }^{2}+k_{z}{ }^{2}\right)$ calculated using the radial-symmetric HNC theory for spherical particles is part of the input data. We emphasize that the $\mathrm{OZ}$ equation is exact. On the other hand, the bridge function is neglected in the HNC closure equation. However, it has been verified that the $3 \mathrm{D}-\mathrm{OZ}-\mathrm{HNC}$ theory gives quantitatively reliable results [12].

The numerical procedure is briefly summarized as follows: (1) $u_{1 \mathrm{~S}}(x, y, z)$ is calculated at each 3D grid point, (2) $w_{1 \mathrm{~S}}(x, y, z)$ is initialized to zero, (3) $c_{1 \mathrm{~S}}(x, y, z)$ is calculated from Eq. (2), and $c_{1 \mathrm{~S}}(x, y, z)$ is transformed to $C_{1 \mathrm{~S}}\left(k_{x}, k_{y}, k_{z}\right)$ using the 3D fast Fourier transform (3D-FFT), (4) $W_{1 \mathrm{~S}}\left(k_{x}, k_{y}, k_{z}\right)$ is calculated from Eq. (1), and $W_{1 \mathrm{~S}}\left(k_{x}, k_{y}, k_{z}\right)$ is inverted to $w_{1 \mathrm{~S}}(x, y, z)$ using the 3D-FFT, and (5) steps (3) and (4) are repeated until the input and output functions for $w_{1 \mathrm{~S}}(x, y, z)$ become identical within convergence tolerance. On grid points where a solvent particle and the solute overlap, $\exp \left\{-u_{1 \mathrm{~S}}(x, y, z) /\left(k_{\mathrm{B}} T\right)\right\}$ is zero. On those where a solvent particle is in contact with the solute, it is set at 0.5 , and otherwise it is unity. The grid spacing ( $\Delta x, \Delta y$, and $\Delta z$ ) is set at $0.1 d_{\mathrm{S}}$, and the grid resolution $\left(N_{x} \times N_{y} \times N_{z}\right)$ is $256 \times 256 \times 256$. It has been verified that the spacing is sufficiently small and the box size $\left(N_{x} \Delta x, N_{y} \Delta y, N_{z} \Delta \mathrm{z}\right)$ is large enough for the correlation functions at the box surfaces to be essentially zero. 
First, the Fourier transform of the vessel-solvent direct correlation function denoted by $C_{1 \mathrm{~S}}\left(k_{x}, k_{y}, k_{z}\right)$ is calculated by following the procedure described above. Second, the Fourier transforms of the solute-solvent total correlation function denoted by $H_{2 S}(k)$ (the subscript " 2 " denotes the solute) is calculated using the radial symmetric HNC theory for spherical particles. The PMF between the vessel and the solute, $\Phi_{12}(x, y, z)$, is then obtained from

$\Phi_{12}(x, y, z) /\left(k_{B} T\right)=u_{12}(x, y, z) /\left(k_{\mathrm{B}} T\right)-w_{12}(x, y, z)$,

where $w_{12}(x, y, z)$ is calculated by inverting $W_{12}\left(k_{x}, k_{y}, k_{z}\right)$ given by

$W_{12}\left(k_{x}, k_{y}, k_{z}\right)=\rho_{\mathrm{S}} C_{1 \mathrm{~S}}\left(k_{x}, k_{y}, k_{z}\right) H_{2 \mathrm{~S}}(k)$.

The physical meaning of $\Phi_{12}(x, y, z)[6,14]$ can be understood from

$\Phi_{12}(x, y, z)=F(x, y, z)-F(\infty, \infty, \infty)$

and

$g_{12}(x, y, z)=\exp \left\{-\Phi_{12}(x, y, z) /\left(k_{\mathrm{B}} T\right)\right\}, g_{12}(\infty, \infty, \infty)=1$.

$F(x, y, z)$ is the free energy of small spheres in the case where the big-sphere center is at the position $(x, y, z)$, and $g_{12}(x, y, z)$ the pair distribution function. For rigid-body models, the behavior of $\Phi_{12}(x, y, z)$ is purely entropic in origin. Due to the microscopic structure of small spheres formed within the domain confined by the big sphere and the vessel, $\Phi_{12}(x, y, z)$ exhibits a complex spatial distribution. A great advantage of the 3D integral equation theory is that the values of $\Phi_{12}$ on all the grid points are obtained from only a single calculation, which is in marked contrast with the usual computer simulation.

We emphasize that the geometry variation is made continuously though it is presented in a stepwise manner in Fig. 1. Figure 1a shows the initial geometry of the vessel, a cylinder with inner diameter $8 d_{\mathrm{S}}$, length $14 d_{\mathrm{S}}$, and thickness $d_{\mathrm{S}}$. These dimensions roughly mimic those of TolC except that the length is set at a much shorter value (the length of TolC is $\sim 35 d_{\mathrm{S}}$ ). This is because all we need in this Letter is a length which is sufficiently longer than the diameter of the solute $d_{\mathrm{B}}$ set at $4 d_{\mathrm{S}}$. The left and right ends of the cylindrical vessel are the entrance and exit, respectively. The inner diameter at the entrance is first reduced to $7.7 d_{\mathrm{S}}$ (Fig. 1b). $L_{1}$ and $L_{2}$ in Fig. 1c (lengths of the portions with inner diameter $7.7 d_{\mathrm{S}}$ and $8 d_{\mathrm{S}}$, respectively) are gradually increased and decreased, respectively. The length of the tapering portion is set at $2 d_{\mathrm{S}}$ $\left(L_{1}+L_{2}=12 d_{\mathrm{S}}\right)$. The PMF for $L_{1}=L_{2}=6 d_{\mathrm{S}}$ is presented in this Letter. After the geometry shown in Fig. 1d, the inner diameter uniformly becomes $7.7 d_{\mathrm{S}}$ (Fig. 1e). As the final geometry, the inner diameter at the exit is further reduced to $7.4 d_{\mathrm{S}}$ (Fig. 1f). Hereafter, the vessel geometries shown in Fig. 1a, b, c, d, e, and $\mathrm{f}$ are referred to as vessel 
geometries a, b, c, d, e, and f, respectively.

\section{Results and Discussion}

\subsection{Entropic potential for vessel geometry a in Fig. 1}

Hereafter, the PMF scaled by $k_{\mathrm{B}} T$ between the vessel and the solute are denoted simply by $-\Phi_{\mathrm{S}} / k_{\mathrm{B}}$. We refer to $-T \Phi_{\mathrm{S}}$ as "entropic potential". The distribution of $-\Phi_{\mathrm{S}} / k_{\mathrm{B}}$ on the cross section of $z=0$ for the initial vessel geometry (see Fig. 1a) is shown in Fig. 2 a. $-\Phi_{\mathrm{S}} / k_{\mathrm{B}}$ becomes lower as the color approaches dark blue, and it becomes higher as the color approaches dark red. The center of the big sphere cannot enter the domain drawn in white. The profile of $-\Phi_{\mathrm{S}} / k_{\mathrm{B}}$ along the $x$-axis $(y=z=0)$ is shown in Fig. $2 \mathrm{~b}$. There are three narrow domains within which $-\Phi_{\mathrm{S}} / k_{\mathrm{B}}$ is negative as indicated in Fig. 2a. In general, it is difficult for the solute to overcome a free-energy barrier well exceeding $k_{\mathrm{B}} T$ [4] (an important exception is described in the second paragraph of Section 3.3). The solute can spontaneously be inserted into domain 2 or domain 3 due to the essentially zero barrier. The solute can also enter domain 1 without difficulty: The trajectory indicated by the black arrow in Fig. 2 a possesses the lowest value of the barrier, $\sim 0.8 k_{\mathrm{B}} T$. However, we postulate that the solute is inserted into domain 3 , the narrow domain around the central axis of the vessel (i.e., the $x$-axis). In the real system, AcrB interacting with TolC at its entrance sends the solute to the central position within the vessel cavity, and our postulation can thus be justified. Once the solute enters domain 3 , it cannot move in the radial direction. At $x / d_{S^{\sim}}-5.5$ (the position of the lowest value of $-\Phi_{\mathrm{S}}$ ), the barrier for the solute to overcome for moving from domain 3 to domain 2 is $\sim 8.5 k_{\mathrm{B}} T$ and that for moving from domain 2 to domain 1 is $\sim 6.7 k_{\mathrm{B}} T$. These barriers remain quite high for $-6<x / d_{\mathrm{S}}<6$. If the vessel geometry is not changed, the solute will be confined within the region of $-6<x / d_{\mathrm{S}}<6$ (see Fig. $2 \mathrm{~b}$ ).

Here, we explain how the stripe pattern of the entropic potential is formed along the $y$-axis as observed in Fig. 2a. When the separation between the nearest solute and vessel inner surfaces, which is denoted by $\eta$, is not sufficiently close to $n d_{\mathrm{S}}(n=0,1,2$, ...), spaces unavailable to the translational displacement of solvent molecules appear as indicated in Fig. 3a. By contrast, in cases of $\eta \sim n d_{\mathrm{S}}$, such unfavorable spaces do not appear and the solvent particles can efficiently be packed within the domain confined between two surfaces as illustrated in Fig. 3b. The configuration in Fig. 3a is entropically unfavorable, while that in Fig. $3 \mathrm{~b}$ is entropically favorable, leading to the stripe pattern formed along the $y$-axis.

\subsection{Entropic potentials for vessel geometries b, $c$, and $d$ in Fig. 1}

The distributions of $-\Phi_{\mathrm{S}} / k_{\mathrm{B}}$ on the cross section of $z=0$ for vessel geometries $\mathrm{b}, \mathrm{c}$, and $d$ (see Fig. 1b-d) are shown in Fig. 4a, b, and c, respectively. The profile of $-\Phi_{\mathrm{S}} / k_{\mathrm{B}}$ along the $x$-axis $(y=z=0)$ for each entropic potential is shown in Fig. 5: Fig. 5a, b, and c 
correspond to Fig. 4a, b, and c, respectively. As shown in Fig. 2a and explained in Fig. $3,-\Phi_{\mathrm{S}} / k_{\mathrm{B}}$ becomes largely positive and largely negative with the periodicity of the molecular diameter of the solvent, $d_{S}=0.28 \mathrm{~nm}$. Therefore, even a variation of the vessel geometry within the scale of $d_{\mathrm{S}}$ leads to a drastic change in $-\Phi_{\mathrm{S}} / k_{\mathrm{B}}$. In the case of the variation illustrated in Fig. $1 \mathrm{~b}-\mathrm{d},-\Phi_{\mathrm{S}} / k_{\mathrm{B}}$ in the narrow domain around the central axis of the vessel turns largely positive for the region with inner diameter $7.7 d_{\mathrm{S}}$. Since this region is gradually lengthened, the solute is driven to move in the right direction along the $x$-axis (see Figs. 4 and 5).

An important point is that the entropic potential does not remain constant: It is time dependent. There are three different time scales: time scales of the solvent motion, variation in the vessel geometry, and solute motion. The time scale of the solvent motion is doubtlessly the fastest. The solvent is practically in equilibrium with the solute-vessel configuration all the time. Here, we assume that the variation of the vessel geometry (that is, the variation of the entropic potential) is relatively faster than the solute motion. The faster variation of the vessel geometry could be realized by structural changes of AcrB caused by the proton motive force. An entropic force, which is given as $-\left\{\partial\left(-T \Phi_{\mathrm{S}}\right) / \partial x\right\}$, continuously acts on the solute in the right direction along the $x$-axis and accelerates its motion during the variation of the vessel geometry. When vessel geometry $\mathrm{d}$ is reached, the solute possesses a considerably high velocity. As a consequence, the solute is capable of overcoming even a barrier significantly exceeding $k_{\mathrm{B}} T$.

\subsection{Entropic potentials for vessel geometries $d$, e, and $f$ in Fig. 1}

The profile of $-\Phi_{\mathrm{S}} / k_{\mathrm{B}}$ along the $x$-axis $(y=z=0)$ for vessel geometry $\mathrm{d}$ is redrawn for $4 \leq x / d_{\mathrm{S}} \leq 12$ in Fig. 6a. The minimum of $-\Phi_{\mathrm{S}} / k_{\mathrm{B}}$ is located at $\left(x / d_{\mathrm{S}}, y / d_{\mathrm{S}}, z / d_{\mathrm{S}}\right)=(7.3,0$, $0)$. The coordinate, $\left(x / d_{\mathrm{S}}, y / d_{\mathrm{S}}, z / d_{\mathrm{S}}\right)=(7,0,0)$, corresponds to the position where the right half of the solute is outside the vessel. It is observed in Fig. 6a that the solute must overcome the barrier $\sim 3.6 k_{\mathrm{B}} T$ to be released to the bulk in the right direction along the $x$-axis. It is not definite if the solute velocity is high enough to overcome this barrier. However, we find the following: As the inner diameter at the exit $D$ decreases as $(\mathrm{d}) \rightarrow(\mathrm{e}) \rightarrow(\mathrm{f})$ in Fig. $1 \mathrm{~d}-\mathrm{f}$ (the values of $D$ are $8 d_{\mathrm{S}}, 7.7 d_{\mathrm{S}}$, and $7.4 d_{\mathrm{S}}$ in vessel geometries $\mathrm{d}$, e, and $\mathrm{f}$, respectively), the solute is driven to move further in the right direction along the $x$-axis and the barrier becomes progressively lower. The profiles of $-\Phi_{\mathrm{S}} / k_{\mathrm{B}}$ along the $x$-axis $(y=z=0)$ for vessel geometries e and $\mathrm{f}$ are shown in Fig. $6 \mathrm{~b}$ and $\mathrm{c}$, respectively. The barriers in vessel geometries e and $\mathrm{f}$ are $\sim 2.0 k_{\mathrm{B}} T$ and $\sim 1.2 k_{\mathrm{B}} T$, respectively. The barrier can further be reduced by decreasing $D$.

The entropic potential is an effective one, which means that it is subject to fluctuation. When the solute has reached the potential minium in Fig. 6c, it must overcome the barrier $\sim 1.2 k_{\mathrm{B}} T$. However, due to the potential fluctuation, the actual barrier is higher than this value at some times and lower at other times. For this reason, the probability for the solute to overcome such an effective barrier is higher than one might expect from the barrier height. Further, we consider rigid-body interactions. 
Potential barriers in such a model system are higher than in the real system. If the vessel wall and the solute are soft, the oscillation of the entropic potential should be weaker than that calculated in this Letter. It is probable that the barrier for the solute to overcome is smaller than in the rigid-body system.

From the above argument, we conclude that the solute can definitely be released to the bulk. The key idea is a continuous variation of the vessel geometry accompanying that of the spatial distribution of the entropic potential. We remark that the solute velocity in the final vessel geometry becomes higher as the vessel length increases. In other words, a longer vessel enables the solute to overcome a higher barrier for being released to the bulk. Even a barrier well exceeding $k_{\mathrm{B}} T$ could be overcome. The very large value of the length of TolC, $\sim 35 d_{\mathrm{S}}$, may play essential roles in the solute release.

\section{Concluding Remarks}

We have investigated insertion and release of a solute into and from a cylindrical vessel possessing an entrance at one end and an exit at the other end for the solute. The entropic potential formed by the solvent is calculated using the $3 \mathrm{D}$ integral equation theory and rigid-body models. With the rigid-body models, all of the allowed system configurations share the same energy, and the system behavior is purely entropic in origin [15]: The insertion/release process is described by the solvent-entropy effect arising from the translational displacement of solvent molecules. It has been demonstrated that the two opposite events, insertion of a solute into the vessel and release of the solute from the vessel, can be driven by the same mechanism. It is quite interesting and important that such rich behavior is observed in a very simple model system.

The results obtained are recapitulated as follows. As long as the vessel geometry is fixed, the entropic component always acts for insertion. We show, however, that a solute which has been inserted can also be released by the solvent-entropy effect using a continuous variation of the vessel geometry. An example of the variation is illustrated in Fig. 1. In the real system, the insertion/release process is to be described in terms of the potential of mean force (PMF) consisting of the energetic and entropic components. The energetic component is strongly dependent on the solute-solvent and vessel inner surface-solvent affinities, whereas the entropic component is rather insensitive to them [6]. The entropic component dominates when the inner surface of the vessel is neither hydrophobic nor hydrophilic. The present study, which shows that both of insertion and release can be achieved solely by the entropic component being uninfluenced by the solute-solvent affinity, is an important first step toward elucidating the microscopic mechanism of the multidrug efflux of TolC [1-3].

To complete a physical picture of the multidrug efflux, we have to find a manner of the entropic release (i.e., a reliable manner of varying the geometric features of the vessel) which can handle a fairly wide range of the solute size. To know how high a 
barrier can be overcome in the final stage of the solute release (see Section 3.3), we need to know dynamic aspects of the entropic release by simulating a biased Brownian motion of the solute in a time-dependent force field. In any case, a continuous variation of the vessel geometry is the key as pointed out in this Letter. The details of the polyatomic structures of the vessel and/or the solute are also important factors. Further, the solvent-entropy effect should play crucially important roles even in the functioning of AcrB, which is to be investigated. Works in these directions are in progress.

\section{Acknowledgment}

This work was supported by Grants-in-Aid for Scientific Research on Innovative Areas (No. 20118004) and that on (B) (No. 22300100) from the Ministry of Education, Culture, Sports, Science and Technology of Japan, by Kyoto University Global Center of Excellence of Energy Science, and by Grant-in-Aid for JSPS (Japan Society for the Promotion of Science) fellows. 


\section{References}

[1] V. Koronakis, A. Sharff, E. Koronakis, B. Luisi, C. Hughes, Nature 405 (2000) 914.

[2] L. Vaccaro, K. A. Scott, M. S. P. Sansom, Biophys. J. 95 (2008) 5681.

[3] K. M. Pos, Biochim. Biophys. Acta 1794 (2009) 782.

[4] K. Amano, M. Kinoshita, Chem. Phys. Lett. 488 (2010) 1.

[5] K. Amano, M. Kinoshita, Chem. Phys. Lett. 504 (2011) 221.

[6] K. Amano, H. Oshima, M. Kinoshita, J. Chem. Phys. 135 (2011) 185101.

[7] A. L. Horwich, W. A. Fenton, E. Chapman, G. W. Farr, Annu. Rev. Cell. Dev. Biol. 23 (2007) 115.

[8] T. K. Chaudhuri, V. K. Verma, A. Maheshwari, Prog. Biophys. Mol. Biol. 99 (2009) 42.

[9] S. Tanaka, Y. Kawata, G. Otting, N. E. Dixon, K. Matsuzaki, M. Hoshino, Biochim. Biophys. Acta 1804 (2010) 866.

[10] D. Beglov, B. Roux, J. Chem. Phys. 103 (1995) 360.

[11] M. Ikeguchi, J. Doi, J. Chem. Phys. 103 (1995) 5011.

[12] M. Kinoshita, J. Chem. Phys. 116 (2002) 3493.

[13] M. Kinoshita, Chem. Phys. Lett. 387 (2004) 47.

[14] K. Amano, T. Yoshidome, M. Iwaki, M. Suzuki, M. Kinoshita, J. Chem. Phys. 133 (2010) 045103.

[15] M. Kinoshita, Chem. Eng. Sci. 61 (2006) 2150.

[16] T. Yoshidome, M. Kinoshita, S. Hirota, N. Baden, M. Terazima, J. Chem. Phys. 128 (2008) 225104.

[17] T. Yoshidome, M. Kinoshita, Phys. Rev. E 79 (2009) 090305(R).

[18] H. Oshima, T. Yoshidome, K. Amano, M. Kinoshita, J. Chem. Phys. 131 (2009) 205102.

[19] Y. Harano, M. Kinoshita, Biophys. J. 89 (2005) 2701.

[20] M. Kinoshita, Front. Biosci. 14 (2009) 3419.

[21] M. Kinoshita, Int. J. Mol. Sci. 10 (2009) 1064.

[22] S. Asakura and F. Oosawa, J. Chem. Phys. 22 (1954) 1255.

[23] S. Asakura and F. Oosawa, J. Polym. Sci. 33 (1958) 183.

[24] P. Attard and G. N. Patey, J. Chem. Phys. 92 (1990) 4970.

[25] M. Kinoshita, S. Iba, K. Kuwamoto, M. Harada, J. Chem. Phys. 105 (1996) 7177.

[26] R. Roth, B. Götzelmann, S. Dietrich, Phys. Rev. Lett. 83 (1999) 448.

[27] M. Kinoshita, Chem. Phys. Lett. 353 (2002) 259.

[28] R. Roth and M. Kinoshita, J. Chem. Phys. 125 (2006) 084910.

[29] P.-M. König, R. Roth, and S. Dietrich, Phys. Rev. E 74 (2006) 041404.

[30] P.-M. König, R. Roth, and S. Dietrich, Europhys. Lett. 84 (2008) 68006. 


\section{Figure Captions}

Fig. 1. Variation of vessel geometry: (a) $\rightarrow($ b) $\rightarrow($ c $) \rightarrow(d) \rightarrow(e) \rightarrow(f)$. The geometry in (a) is a cylinder with inner diameter $8 d_{\mathrm{S}}$, length $14 d_{\mathrm{S}}$, and thickness $d_{\mathrm{S}}$. The coordinate system is chosen as illustrated here. The cross section of $z=0$ is shown for each geometry. Though the geometry variation is illustrated in a stepwise manner in this figure, it is made continuously.

Fig. 2. (a) Distribution of $-\Phi_{\mathrm{S}} / k_{\mathrm{B}}$ on the cross section of $z=0$ for the initial vessel geometry shown in Fig. 1a. $-\Phi_{\mathrm{S}} / k_{\mathrm{B}}$ becomes lower as the color approaches dark blue, and it becomes higher as the color approaches dark red ("max" and "min" represent the maximum and minimum values, respectively). The center of the large sphere cannot enter the domain drawn in white. " 0.8 " represents that the free-energy barrier along the black arrow is $\sim 0.8 k_{\mathrm{B}} T$. (b) Profile of $-\Phi_{\mathrm{S}} / k_{\mathrm{B}}$ along the $x$-axis $(y=z=0)$ for the initial vessel geometry shown in Fig. 1a. The two broken lines represent positions of the vessel ends, $x / d_{\mathrm{S}}= \pm 7$.

Fig. 3. Cartoons illustrating how the stripe pattern of the entropic potential shown in Fig. 2a is formed along the $y$-axis. (a) Separation between the nearest solute and vessel inner surfaces, which is denoted by $\eta$, is not sufficiently close to $n d_{\mathrm{S}}(n=0,1,2, \ldots)$. (b) In the case of $\eta \sim n d_{\mathrm{S}}$.

Fig. 4. (a) Distribution of $-\Phi_{\mathrm{S}} / k_{\mathrm{B}}$ on the cross section of $z=0$ for the vessel geometry shown in Fig. 1b. (b) That for the vessel geometry shown in Fig. 1c (both of $L_{1}$ and $L_{2}$ are set at $6 d_{\mathrm{S}}$ ). (c) That for the vessel geometry shown in Fig. 1d. $-\Phi_{\mathrm{S}} / k_{\mathrm{B}}$ becomes lower as the color approaches dark blue, and it becomes higher as the color approaches dark red ("max" and "min" represent the maximum and minimum values, respectively). The center of the large sphere cannot enter the domain drawn in white.

Fig. 5. (a) Profile of $-\Phi_{\mathrm{S}} / k_{\mathrm{B}}$ along the $x$-axis $(y=z=0)$ for the entropic potential shown in Fig. 4a. (b) That shown in Fig. 4b. (c) That shown in Fig. 4c. The two broken lines in each plot represent positions of the vessel ends, $x / d_{\mathrm{S}}= \pm 7$.

Fig. 6. (a) Profile of $-\Phi_{\mathrm{S}} / k_{\mathrm{B}}$ along the $x$-axis $(y=z=0)$ for the vessel geometry shown in Fig. 1d. (b) That for the vessel geometry shown in Fig. 1e. (c) That for the vessel geometry shown in Fig. 1f. The broken line in each plot represents position of the right end of the vessel, $x / d_{\mathrm{S}}=7$. 
Fig. 1

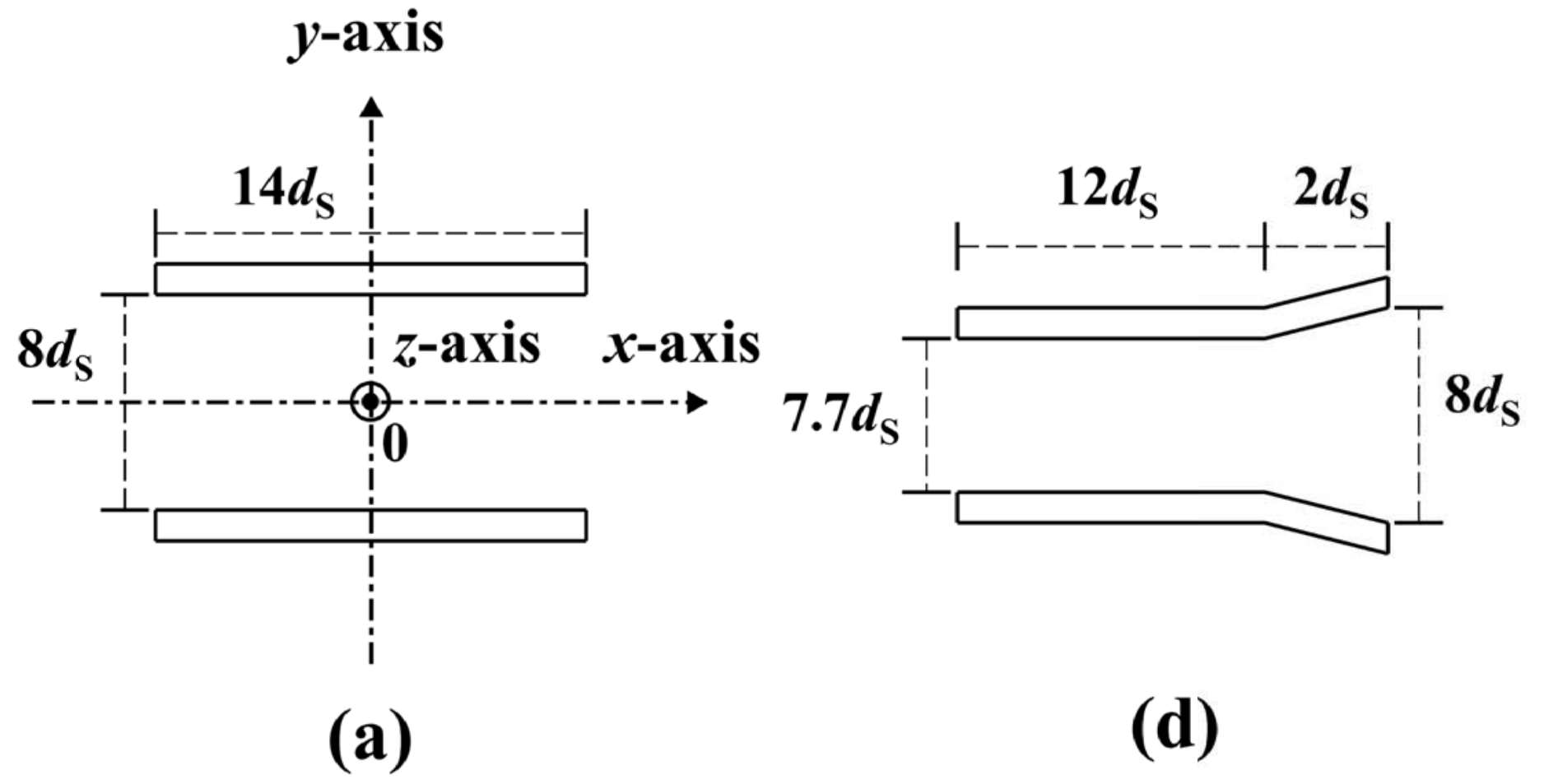

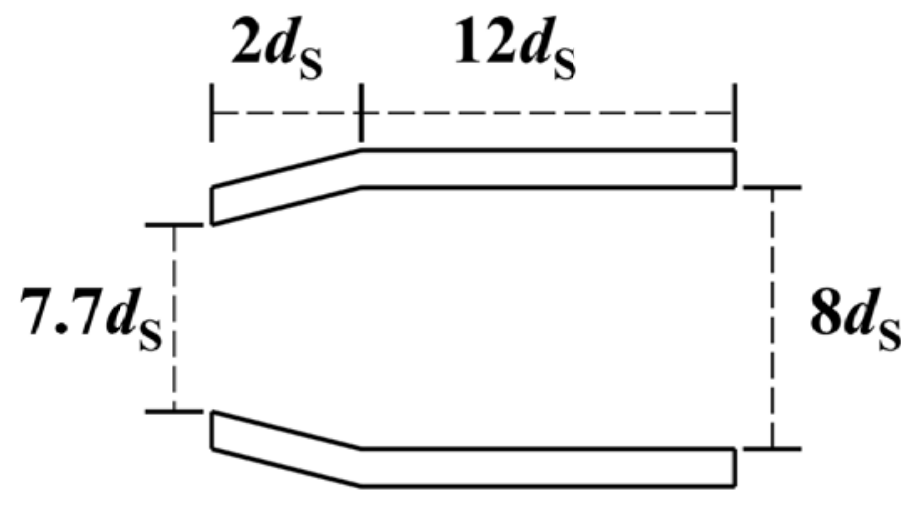

(b)

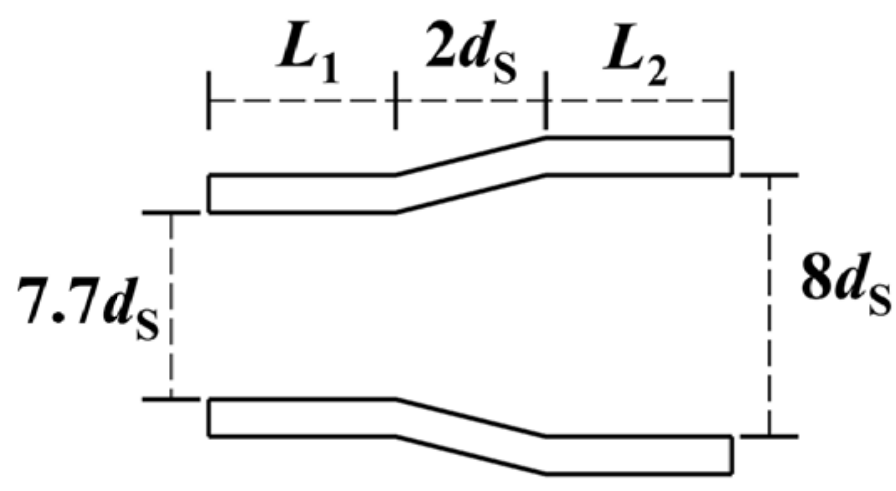

(c)

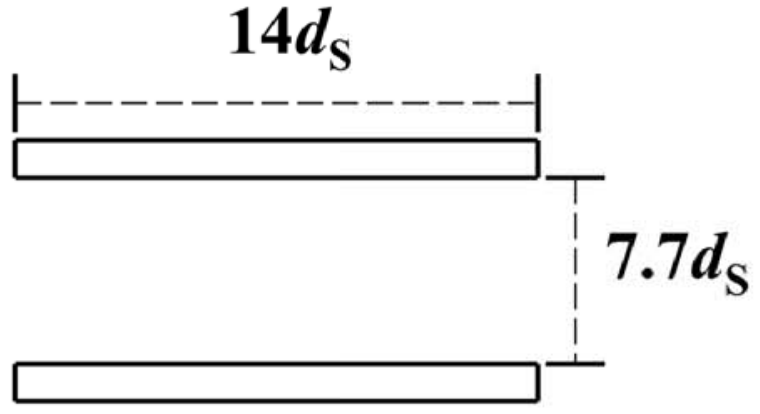

(e)
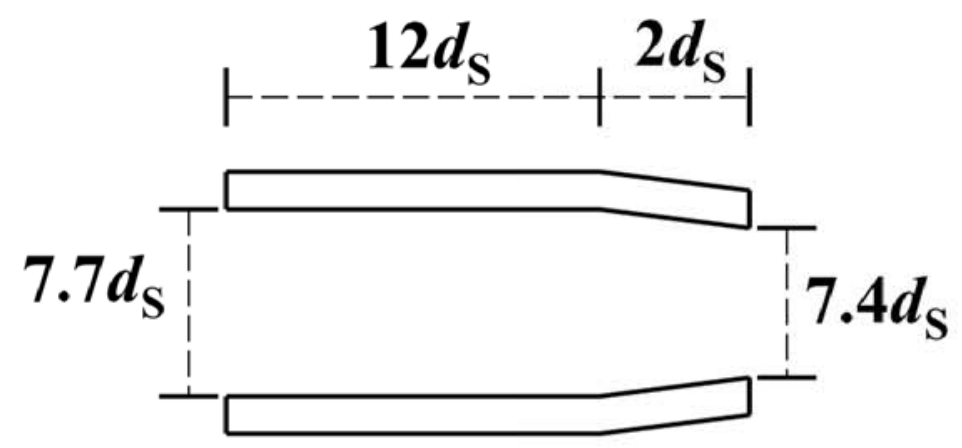

(f) 
Fig. 2
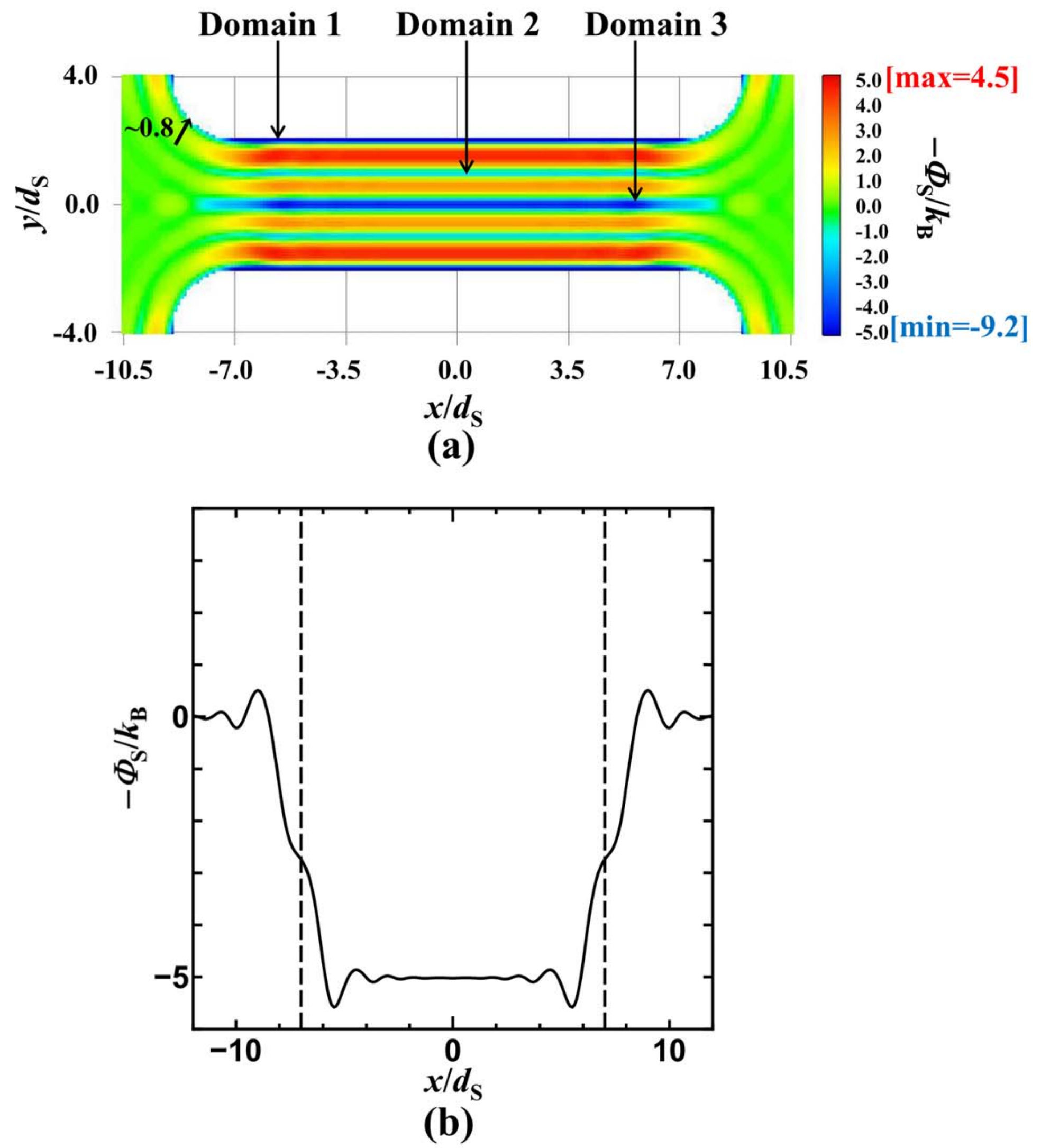
Fig. 3

Spaces unavailable to the translational displacement of solvent molecules
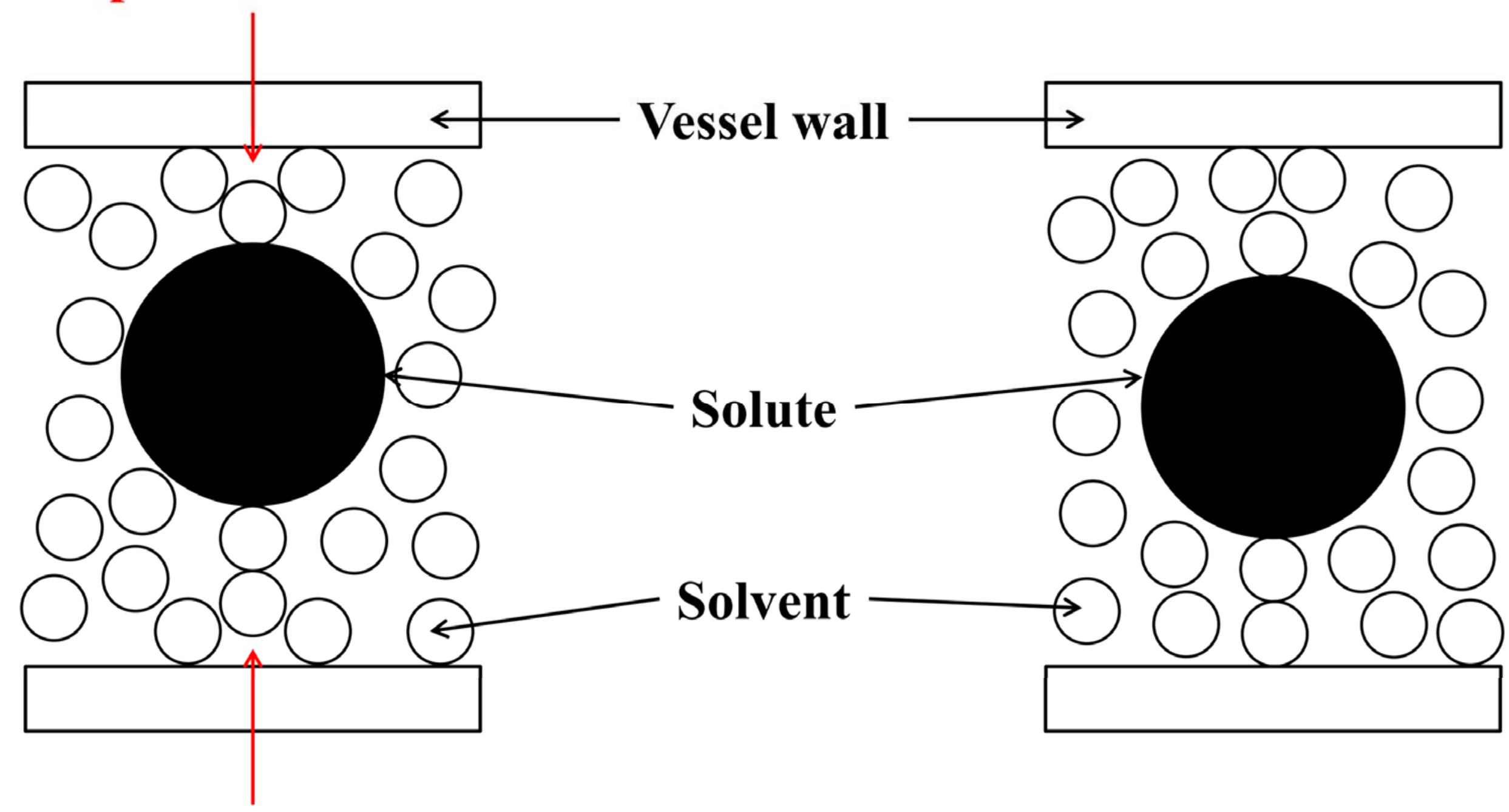

(a)

(b) 
Fig. 4
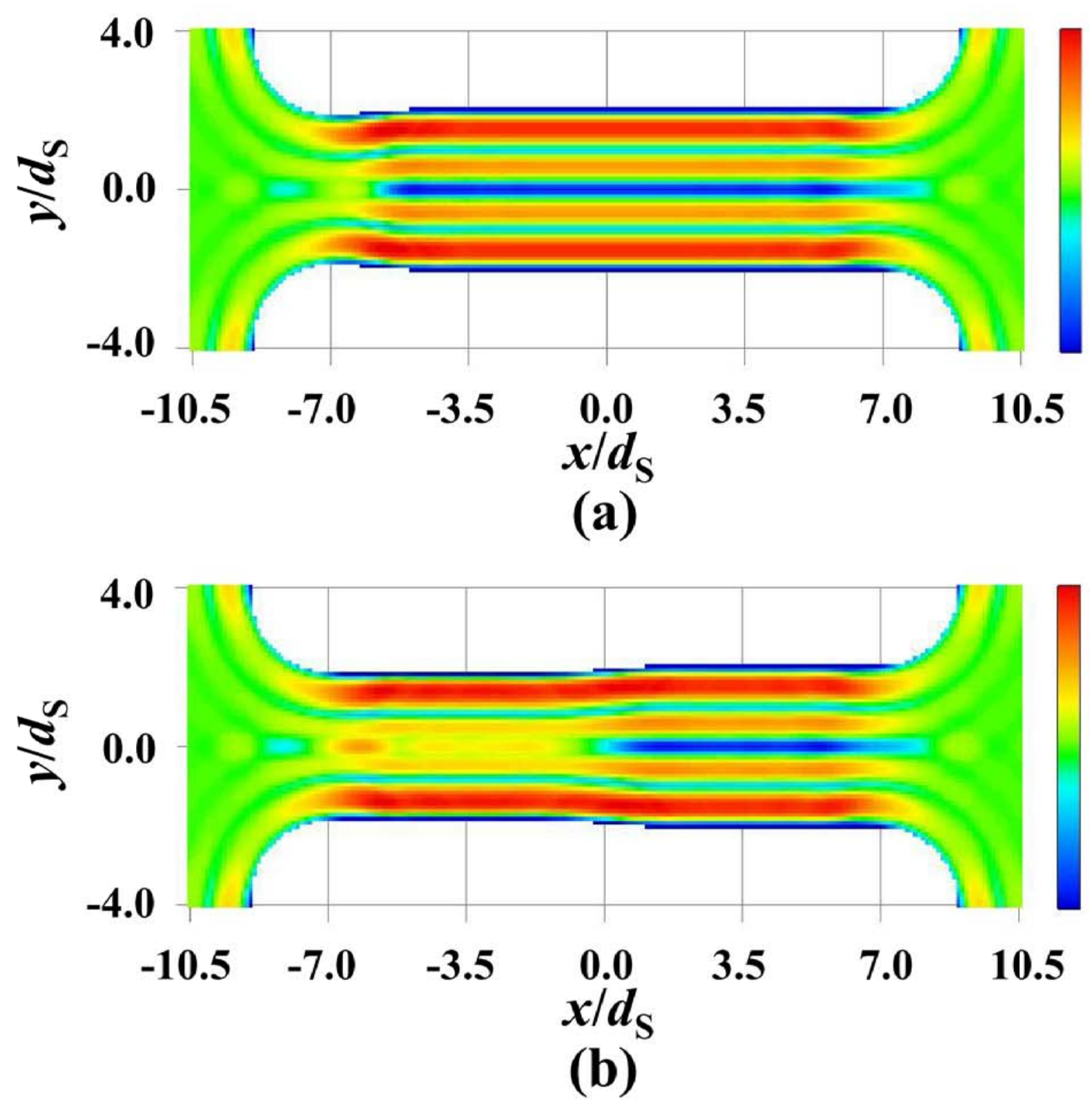

5.0 $[\max =5.0]$

3.0
2.0

1.0

0.0

$-1.0$

-2.0 हा

$\mathbf{- 3 . 0}$

$-4.0$

$-5.0[\mathrm{~min}=-9.2]$

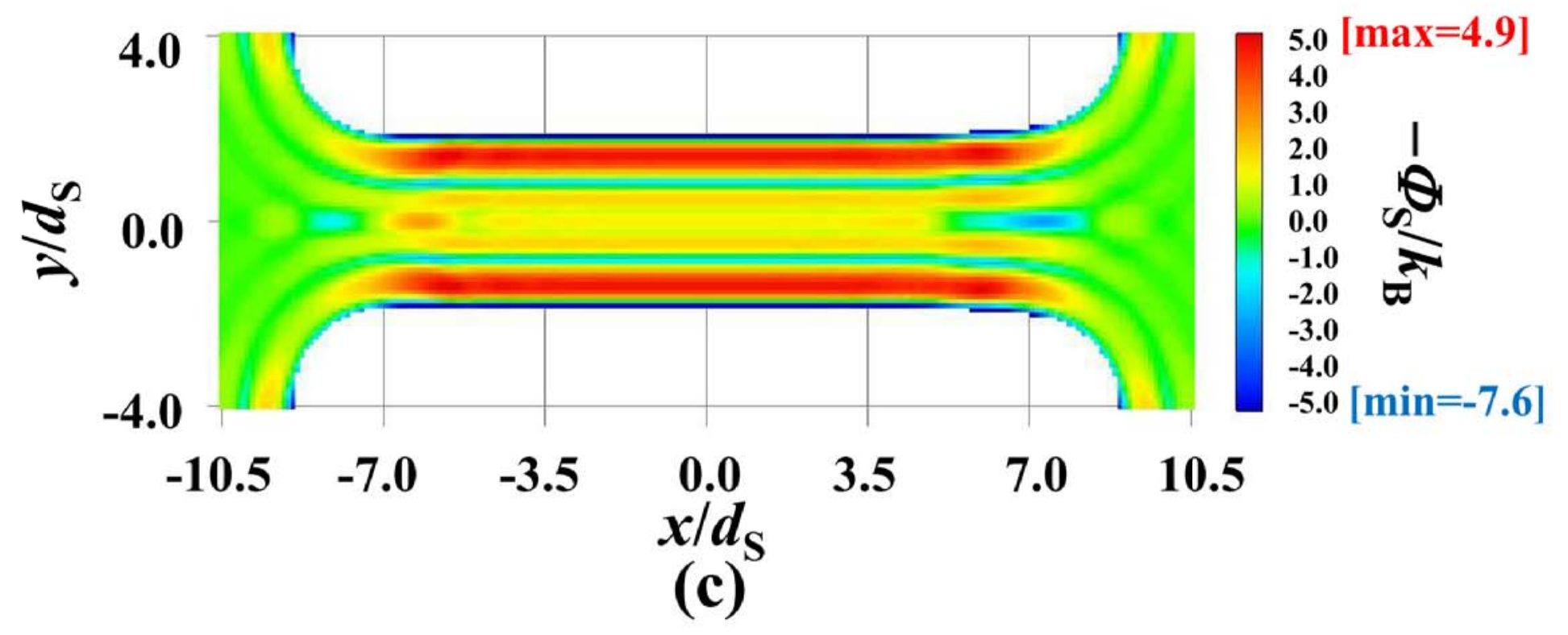


Fig. 5

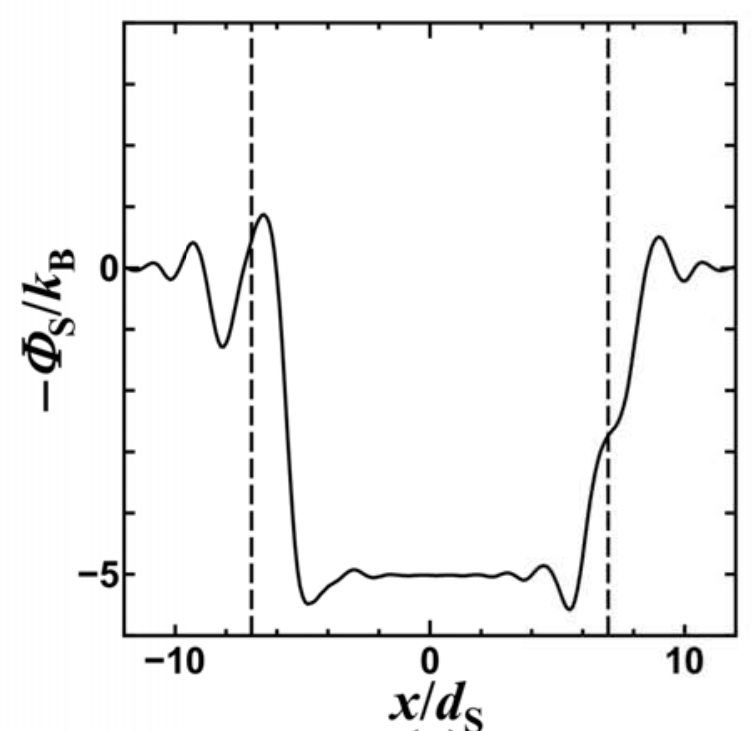

(a)

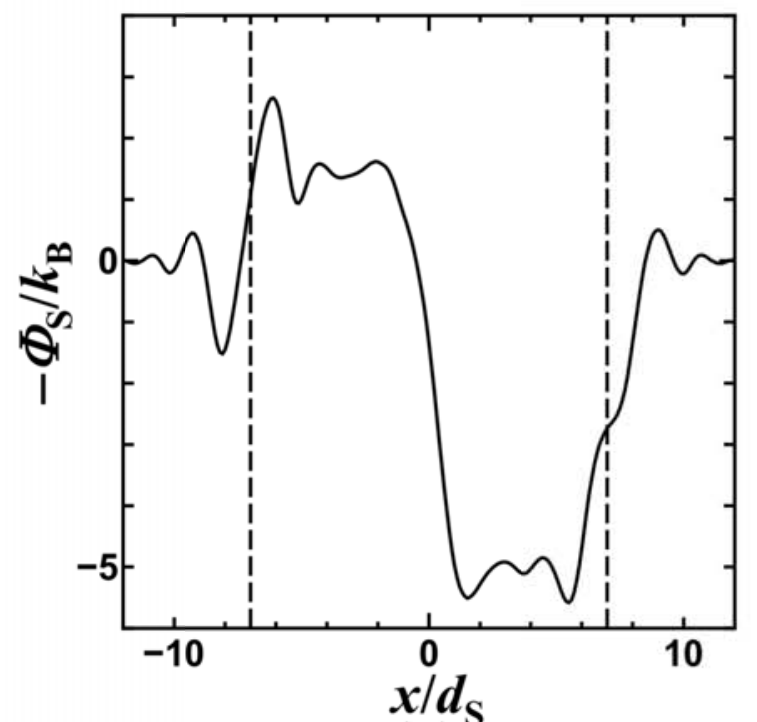

(b)

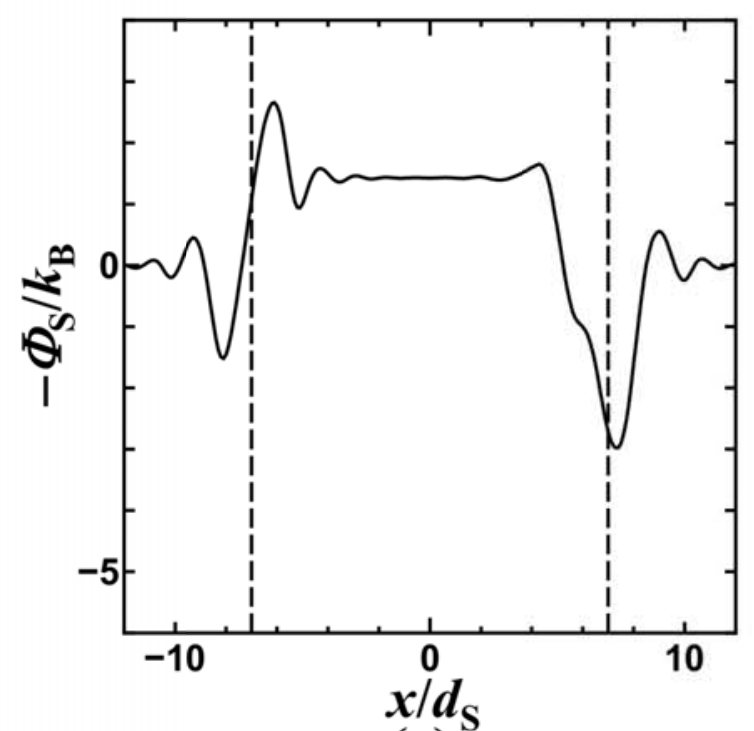

(c) 
Fig. 6

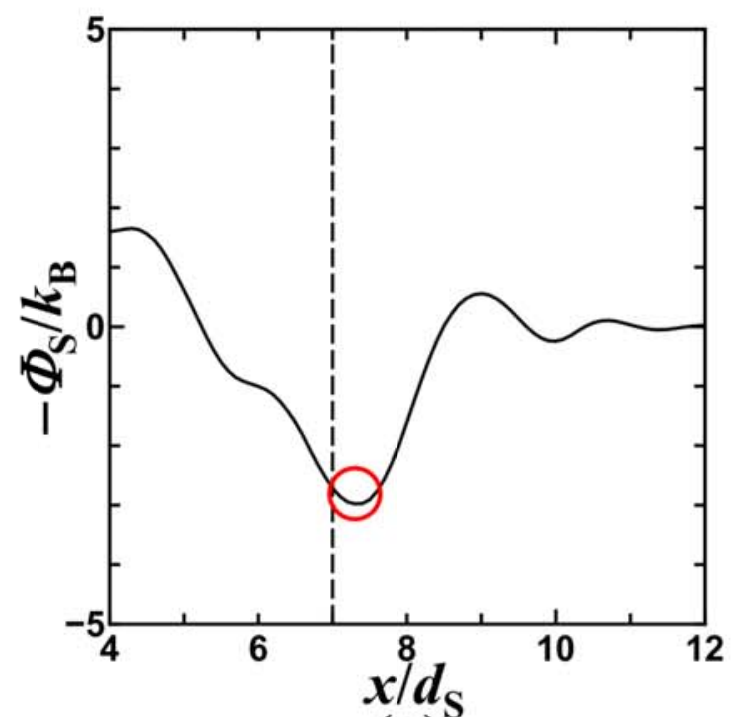

(a)
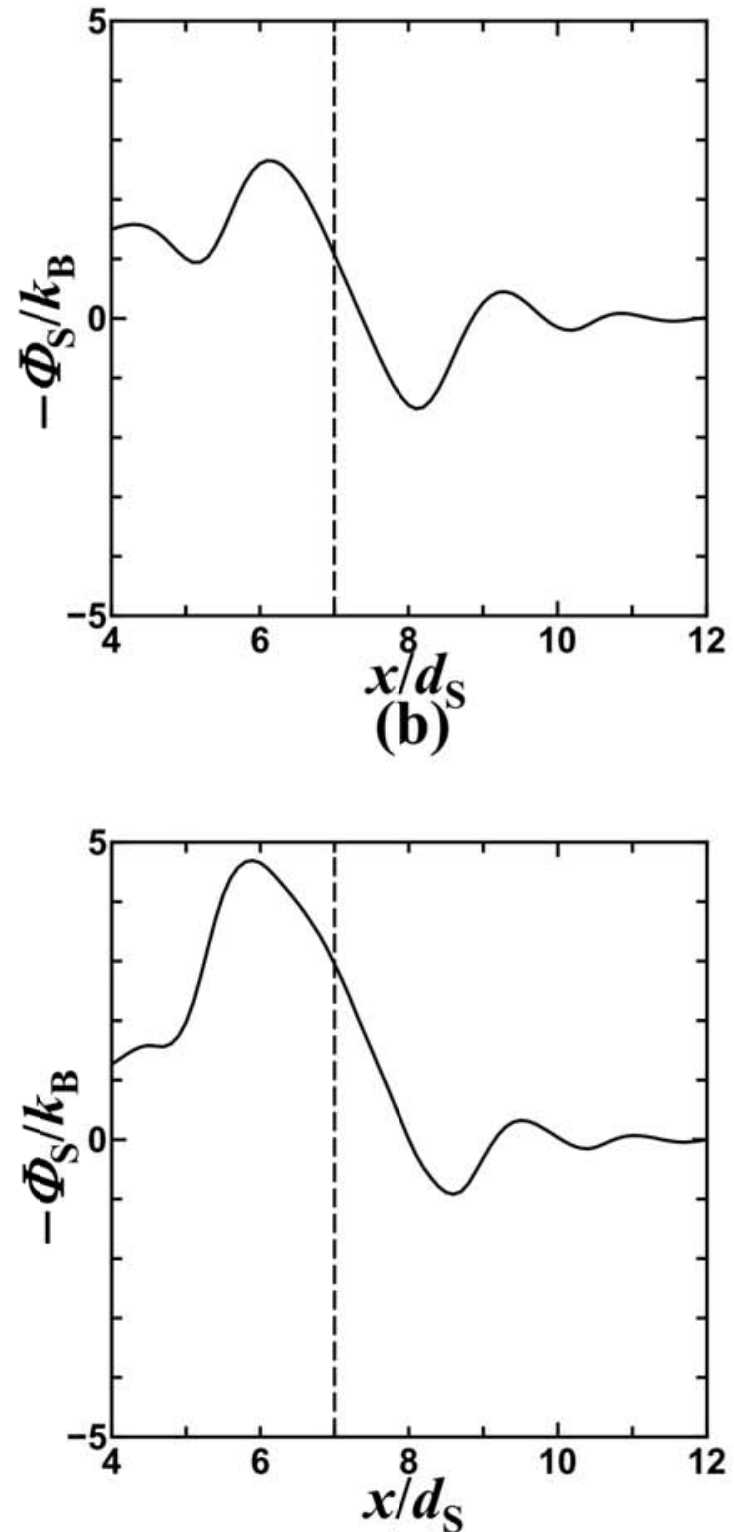

(c) 